\title{
Cine magnetic resonance urography and Whitaker test: dynamic visualized and quantified tools in ileal ureter replacement
}

\author{
Xinfei $\mathrm{Li}^{1 \#}$, Xiang Wang ${ }^{1 \#}$, Teng $\mathrm{Li}^{1 \#}$, Weijie Zhu ${ }^{1}$, Mingming $\mathrm{Ma}^{2}$, Kunlin Yang ${ }^{1}$, Zhihua $\mathrm{Li}^{1}, \mathrm{Juan} \mathrm{Wu}^{3}$, \\ He Wang ${ }^{2}$, Limin Liao ${ }^{3}$, Xuesong Li $^{1}$, Liqun Zhou ${ }^{1}$ \\ ${ }^{1}$ Department of Urology, Peking University First Hospital, Institute of Urology, Peking University, National Urological Cancer Center, Beijing, \\ China; ${ }^{2}$ Department of Radiology, Peking University First Hospital, Beijing, China; ${ }^{3}$ China Rehabilitation Research Center, Beijing Boai Hospital, \\ Beijing, China \\ Contributions: (I) Conception and design: X Li, J Wu, H Wang; (II) Administrative support: L Liao, L Zhou; (III) Provision of study materials or \\ patients: W Zhu, M Ma, Z Li; (IV) Collection and assembly of data: X Li, X Wang, K Yang; (V) Data analysis and interpretation: X Li, X Wang, K \\ Yang; (VI) Manuscript writing: All authors; (VII) Final approval of manuscript: All authors. \\ "These authors contributed equally to this work. \\ Correspondence to: Xuesong Li. Department of Urology, Peking University First Hospital, Institute of Urology, Peking University, National Urological \\ Cancer Center, No. 8 Xishiku Street, Xicheng District, Beijing 100034, China. Email: pineneedle@sina.com; Limin Liao. China Rehabilitation \\ Research Center, Beijing Boai Hospital, No. 10 Jiaomen North Road, Fengtai District, Beijing 100068, China. Email: limin64@gmail.com; He Wang. \\ Department of Radiology, Peking University First Hospital, Institute of Urology, Peking University. National Urological Cancer Center, No. 8 \\ Xishiku Street, Xicheng District, Beijing 100034, China. Email: wanghecrane@126.com.
}

Background: To assess the feasibility and usefulness of cine magnetic resonance urography (MRU) and Whitaker test as postoperative evaluation tools of ileal ureter replacement.

Methods: We retrospectively collected the medical records of 42 patients who underwent ileal ureter replacement between August 2015 and August 2020. The morphology, luminal diameter, amplitude, contraction ratio, peristaltic frequency, ureteral jets and peristalsis efficiency were recorded in cine MRU. Under different perfusion loads, image and pressure changes of the reconstructed upper urinary tract were recorded in the Whitaker test. Patients were categorized into normal pressure and elevated pressure groups. Results: A total of 42 patients underwent cine MRU, and 20 of them finished the Whitaker test successfully. The mean amplitude was $9.29 \pm 3.51 \mathrm{~mm}$, and the contraction ratio was $0.607(0.247-0.790)$. The median peristaltic efficiency was $0.75(0.29-1)$. Three patients presented an unusual rise in renal pelvis pressure, which was $54,26,57 \mathrm{cmH}_{2} \mathrm{O}$ respectively. The amplitude of the ileal graft in the elevated pressure group was larger $(13.80 \pm 5.73$ vs. $8.09 \pm 3.38 \mathrm{~mm}, \mathrm{P}=0.024)$, the contraction ratio was higher $[0.68(0.59-0.79)$ vs. 0.59 (0.25-0.79), $\mathrm{P}=0.028$ ], the peristaltic frequency was more active [7 (6-8) vs. 4 (3-8), $\mathrm{P}=0.025$, but the peristaltic efficiency was lower $[0.50(0.29-0.50)$ vs. $0.75(0.33-1), \mathrm{P}=0.029]$ compared to the normal pressure group. There were no significant differences in the ureteral jets [3 (2-4) vs. $3(1-7), \mathrm{P}=0.840)$, creatinine $(97.3 \pm 7.3$ vs. $103.2 \pm 30.7 \mu \mathrm{mol} / \mathrm{L}, \mathrm{P}=0.753)$, and estimated glomerular filtration rate (eGFR) $(76.4 \pm 14.1$ vs. $\left.68.5 \pm 28.7 \mathrm{~mL} / \mathrm{min} \cdot 1.73 \mathrm{~m}^{2}, \mathrm{P}=0.663\right)$.

Conclusions: Cine MRU provides morphological and peristaltic motility of the ileal graft, cine MRU after ileal ureter replacement was recommended as a routine examination. The Whitaker test represents a complementary investigation to evaluate pressure changes to reveal the ability to transport urine, and it can be used as a supplementary examination to clarify equivocal cases.

Keywords: Ureteral stricture; reconstruction; ileum; imaging; magnetic resonance

Submitted Jun 05, 2021. Accepted for publication Sep 18, 2021.

doi: $10.21037 /$ tau-21-507

View this article at: https://dx.doi.org/10.21037/tau-21-507 


\section{Introduction}

Long segment ureteric lesions present complex challenges to urological surgeons. Ileal ureter replacement is regarded as the last option for more extensive stenosis that is difficult to reconstruct by other techniques, particularly for fulllength defects $(>20 \mathrm{~cm})(1)$. Reestablishing the ureteral continuity by ileal graft presents a technical challenge to urologists. Intestine-related complications after surgery are complex and even life-threatening $(2,3)$. Therefore, follow-up is essential in ileal ureter replacement. Patients are usually advised to receive abdominal ultrasonography, diuretic renography, computed tomography (CT), or magnetic resonance urography (MRU) at the time of follow-up (4).

Compared to other imaging examinations, MRU can not only provide high-contrast resolution imaging but also prevent patients from contrast media and ionizing radiation (5). However, present imaging examinations are quiescent, which could not intuitively show peristalsis and patency of the ileal graft. Differing from conventional MRU, cine MRU allows for continuous and dynamic visualization (6). Such an emerging imaging technique may play an important role in evaluating reconstructed urinary tract function and detecting abnormalities.

In addition to assessing the function of the ileal graft in cine MRU, the Whitaker test, another dynamic examination, can measure pressure changes under given perfusion rates and assess the tolerance of reconstructed upper urinary tract (7). It has been proved useful in judging ambiguous obstruction due to equivocal results from other tests (8).

The purpose of this study is to present our initial experience in using the cine MRU and Whitaker test to evaluate morphology, motility, urination function after ileal ureter replacement. We resent the following article in accordance with the STROBE reporting checklist (available at https://dx.doi.org/10.21037/tau-21-507).

\section{Methods}

\section{Patients}

We conducted a retrospective study of ileal ureter replacement in 42 patients from August 2015 to August 2020. All the surgeries were performed by the same surgeon, and the technique was reported in the previous study (9). Data on the patients' characteristics, stricture length, symptoms, degree of hydronephrosis, preoperative drainage methods, laboratory tests, length of the ileal graft, and follow-up information were collected from our RECUTTER (Reconstruction of Urinary Tract: Technology, Epidemiology, and Result) database. This study was approved by the ethics committee of our hospital (No. 2020-SR-283). Informed consent was obtained from all individual participants included in the study. All research procedures were conducted in accordance with the Declaration of Helsinki (as revised in 2013).

\section{Protocol}

The Whitaker test was performed before removing a nephrostomy catheter at 3 months after the surgery with reference to previous research (10). Nephrostomy tubes and preliminarily retained urinary catheters were connected to the pressure transducer respectively. The pressure was recorded from the renal pelvis and the bladder. X-ray was simultaneously used to evaluate the morphology of ileal ureter. Cine MRU was performed at 3-6 months after the surgery when postoperative protective drainage was removed. The patient needs to hold back urine for cine MRU examination. Static T2-weighted half-Fourier acquisition single-shot turbo spin-echo (HASTE) sequence images, which covered the entire upper urinary tract, were obtained. The specific parameters of HASTE sequence were described in our previous article (11).

\section{Data collection}

Image analysis was performed using a Picture Achieving and Communication System (PACS) workstation by two experienced radiologists. The length of the ileal ureter was measured from pelvis/proximal ureter-ileum anastomosis to ileum-bladder by curving tool in the PACS workstation. The diameter of the upper, middle, lower sites of the ileal graft was measured perpendicular to the direction of the bowel at the maximum extension and the maximum contraction. The amplitude is calculated by subtracting the diameter at maximum contraction from that at maximum extension. The contraction ratio is defined as the amplitude divided by the diameter at the maximum extension. The frequency of both peristaltic waves of the ileal graft and ureteral jets during the whole MRU loop were recorded. The peristalsis efficiency is defined as the ureteral jets divided by peristaltic waves of ileal graft.

Perfusion rate, perfusion volume, renal pelvis pressure, pressure difference, and imaging results were recorded in 
Table 1 The characteristics of the patients

\begin{tabular}{|c|c|}
\hline Variables & Value \\
\hline \multicolumn{2}{|l|}{ Gender, n (\%) } \\
\hline Male & $16(38.1)$ \\
\hline Female & $26(61.9)$ \\
\hline Age (years) & $44.2 \pm 11.5$ \\
\hline $\mathrm{BMI}\left(\mathrm{kg} / \mathrm{m}^{2}\right)$ & $23.2 \pm 2.0$ \\
\hline \multicolumn{2}{|l|}{ Side, n (\%) } \\
\hline Left & $15(35.7)$ \\
\hline Right & $16(38.1)$ \\
\hline Bilateral & $11(26.2)$ \\
\hline Stricture length $(\mathrm{cm})$ & $12.2 \pm 6.2$ \\
\hline \multicolumn{2}{|l|}{ Symptoms, n (\%) } \\
\hline Flankpain & $14(33.3)$ \\
\hline Fever & $6(14.3)$ \\
\hline Nausea or vomit & $6(14.3)$ \\
\hline Hematuria & $4(9.5)$ \\
\hline Asymptomatic & $2(4.8)$ \\
\hline Unknown & $10(23.8)$ \\
\hline \multicolumn{2}{|l|}{ Degree of hydronephrosos, $n(\%)$} \\
\hline Mild & $19(45.2)$ \\
\hline Moderate & $17(40.5)$ \\
\hline Severe & $4(9.5)$ \\
\hline No & $2(4.8)$ \\
\hline \multicolumn{2}{|l|}{ Drainage methods, $\mathrm{n}(\%)$} \\
\hline Double "J" stent & $1(2.4)$ \\
\hline Nephrostomy tube & $33(78.6)$ \\
\hline Double "J" stent history & $20(47.6)$ \\
\hline No & $8(19.0)$ \\
\hline Preoperative creatinine (mmol/L) & $88.7 \pm 26.3$ \\
\hline Preoperative eGFR $\left(\mathrm{mL} / \mathrm{min} \cdot 1.73 \mathrm{~m}^{2}\right)$ & $77.0 \pm 23.8$ \\
\hline
\end{tabular}

eGFR, estimated glomerular filtration rate.

the Whitaker test. It was considered abnormal when there was an unusual rise in renal pelvis pressure higher than $22 \mathrm{cmH}_{2} \mathrm{O}$ during perfusion or a final pressure difference greater than $15 \mathrm{cmH}_{2} \mathrm{O}$. Patients were categorized into normal pressure and elevated pressure groups.

\section{Follow-up}

The follow-ups of patients were managed at 1, 3, and 6 months after surgical treatment and at least once half a year afterward. The patients regularly received symptoms evaluation, physical examinations, urine routine test, blood tests (including serum creatinine, electrolyte test and blood gas analysis) and ultrasound at each visit. Cine MRU and Whitaker tests were performed as above.

\section{Statistical analysis}

All analyses were performed with SPSS ${ }^{\circledast}$ Statistics, version 24.0 (IBM Corp., Armonk, NY, USA). Continuous variables were presented by average \pm standard deviation (age, body mass index, stricture length, creatinine, estimated glomerular filtration rate (eGFR), diameter, amplitude) or median with range (contraction ratio, peristaltic frequency, ureteral jets, peristalsis efficiency). Categorical variables were presented by quantity and percentage (gender, side, symptoms, degree of hydronephrosis, drainage methods). Whether variables were in accordance with normal distribution was checked by the Kolmogorov-Smirnov test. A paired $t$-test was used to compare the difference of average value (creatinine and eGFR) before and after ileal ureter replacement. Differences between the elevated pressure and normal pressure groups were analyzed by either independent sample $t$-test for normally distributed variables (amplitude) or Mann-Whitney $U$ test for nonnormally distributed variables (contraction ratio, peristaltic frequency, ureteral jets and peristaltic efficiency). A twosided $\mathrm{P}<0.05$ was taken to indicate statistical significance.

\section{Results}

A total of 42 patients underwent cine MRU, and 20 of them finished the Whitaker test successfully. The characteristics were shown in Table 1. The median length of the ureteral stricture was $10.0(5.0-30.0) \mathrm{cm}$. Thirty-one patients underwent unilateral ileal ureter replacement, and eleven patients underwent bilateral ileal ureter replacement. All surgeries were completed successfully.

The mean length of ileal ureter was $21.18 \pm 4.92 \mathrm{~mm}$. No leakage was observed in all the cases. All patients showed mild dilatation of the renal pelvis and calyces. The diameter of the upper, middle, lower sites of the ileal graft was measured at the maximum extension and the 

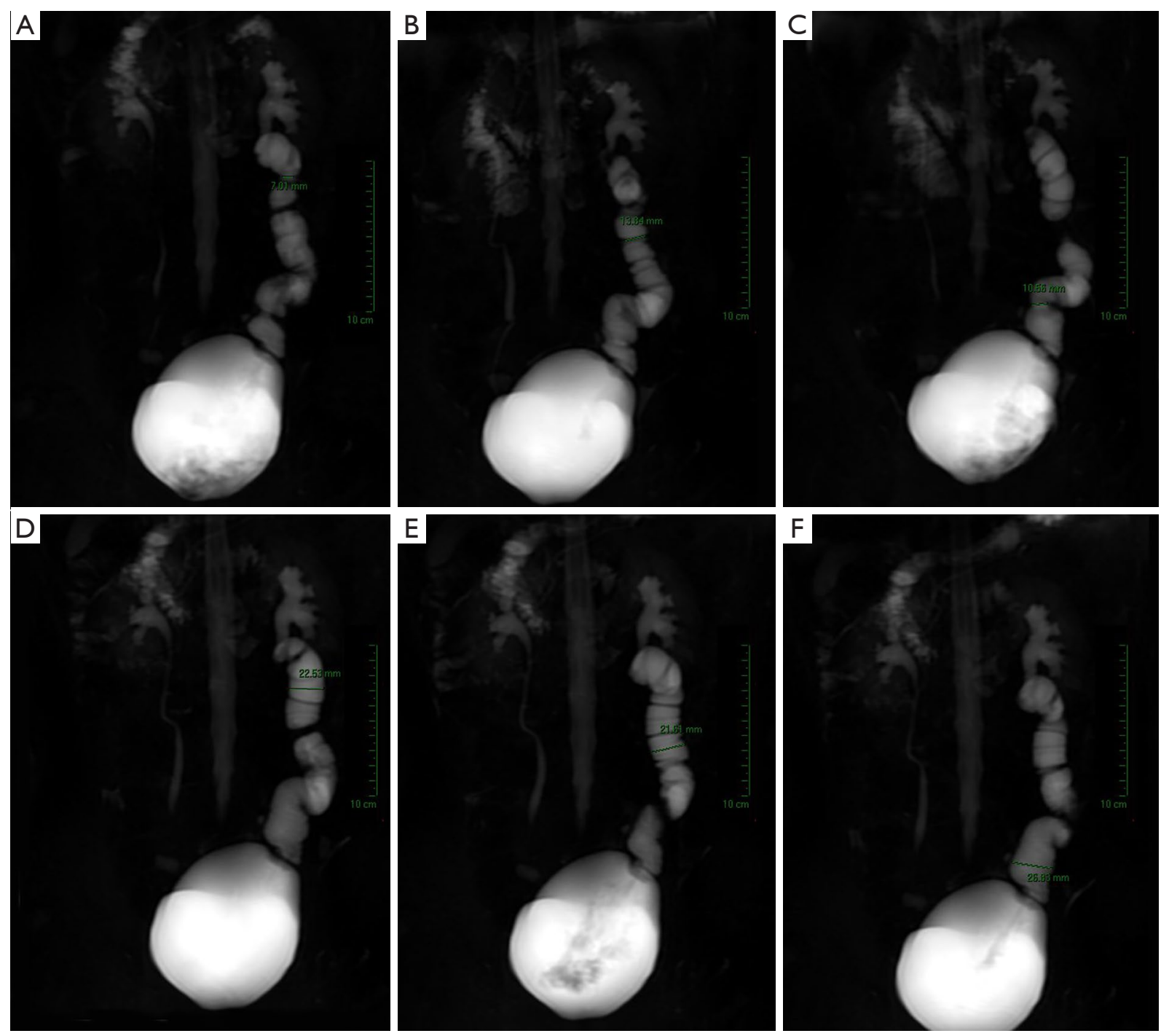

Figure 1 The diameter of upper, middle, lower sites of the ileal graft at the maximum extension and the maximum contraction. (A) Upper, contraction; (B) middle, contraction; (C) lower, contraction; (D) upper, extension; (E) middle, extension; (F) lower, extension.

maximum contraction (Figure 1). The mean amplitude was $9.29 \pm 3.51 \mathrm{~mm}$, and the contraction ratio was 0.607 (0.247-0.790). Ureter-ileum anastomosis, anti-reflux nipple and urine flow were visible (Figures 2,3, see Videos 1,2 for more information). The median peristalsis efficiency was $0.75(0.29-1)$. All the peristaltic parameters of ileal graft in cine MRU did not correlate with postoperative creatinine or eGFR.

The Whitaker test was finished without any perfusionrelated symptoms in 20 patients. Seventeen patients showed no abnormalities in both pressure and imaging during the whole perfusion (Videos 3,4). Among the 17 patients with normal perfusion results, four patients had a negative pressure difference that was lower than $0 \mathrm{cmH}_{2} \mathrm{O}$. Three patients presented an unusual rise in renal pelvis pressure, which was $54,26,57 \mathrm{cmH}_{2} \mathrm{O}$ respectively. Further perfusion showed that the reconstructed upper urinary tract was undeveloped until perfusing to $86 \mathrm{~mL}$ and had insufficient visualization at the ureterovesical junction in one patient (Figure 4). The pressure difference was still higher than $15 \mathrm{mmHg}$ at the end of the perfusion in this patient $(40 \mathrm{mmHg})$. This patient underwent secondary ureteral stent placement.

Patients in the elevated pressure group had larger amplitude 

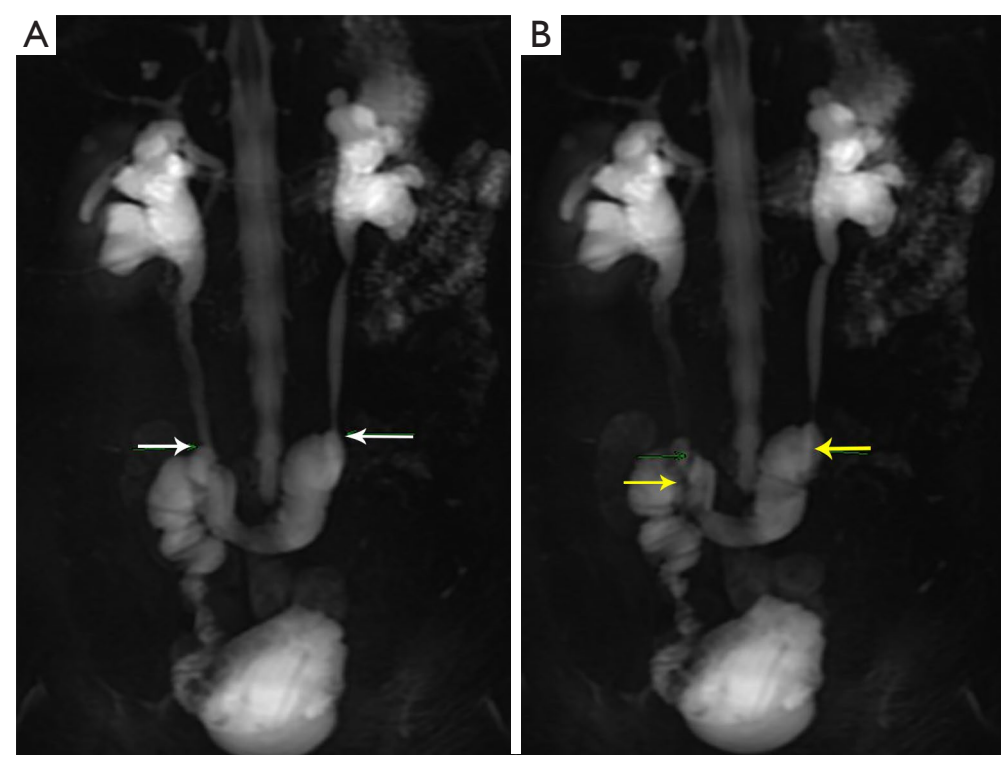

Figure 2 Ureter-ileum anastomosis and urine flow in cine MRU. (A) White arrow, ureter-ileum anastomosis; (B) yellow arrow, urine flow from anastomosis. MRU, magnetic resonance urography.

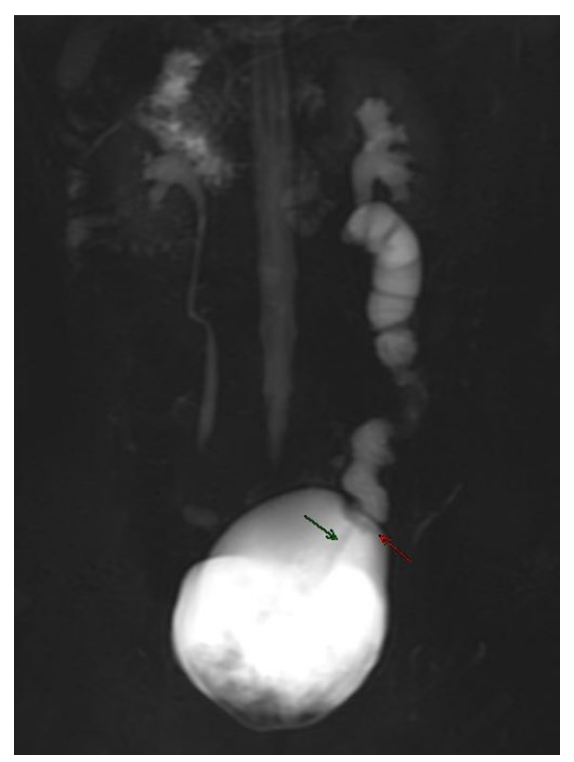

Figure 3 Anti-reflux nipple and ureteral jets in cine MRU. Red arrow, anti-reflux nipple; green arrow, ureteral jets. MRU, magnetic resonance urography.

$(13.80 \pm 5.73$ vs. $8.09 \pm 3.38, \mathrm{P}=0.024)$, higher contraction ratio [0.680 (0.590-0.790) vs. $0.590(0.250-0.790), \mathrm{P}=0.028]$, more peristaltic waves [7 (6-8) vs. 4 (3-8), $\mathrm{P}=0.025]$, but lower peristaltic efficiency $[0.500(0.290-0.500)$ vs. $0.750(0.330-1)$, $\mathrm{P}=0.029]$ compared to the normal pressure group. There were no significant differences in the ureteral jets $[3(2-4) v s$. 3 (1-7), $\mathrm{P}=0.840$ ], creatinine $(97.3 \pm 7.3$ vs. $103.2 \pm 30.7 \mu \mathrm{mol} / \mathrm{L}$, $\mathrm{P}=0.753)$, and eGFR $\left(76.4 \pm 14.1\right.$ vs. $68.5 \pm 28.7 \mathrm{~mL} / \mathrm{min} \cdot 1.73 \mathrm{~m}^{2}$, $\mathrm{P}=0.663)$. The detailed peristaltic information in cine MRU was shown in Table 2. The detailed quantification and imaging results of cine MRU and Whitaker test in each patient were shown in Table S1.

During an average follow-up of $22.2 \pm 15.6$ months, the symptoms remission rate was $95.2 \%$. The postoperative creatinine and eGFR were $95.8 \pm 24.3 \mu \mathrm{mol} / \mathrm{L}$ and $76.6 \pm 24.2 \mathrm{~mL} / \mathrm{min} \cdot 1.73 \mathrm{~m}^{2}$. Renal function remained stable $\left(\mathrm{P}_{\text {crea }}=0.082, \mathrm{P}_{\text {eGFR }}=0.566\right)$.

\section{Discussion}

Ileal ureter replacement is considered as an alternative for long or multiple ureteral defects that are not amenable to be reconstructed by simple means. The successful reconstruction requires not only precise and individualized preoperative planning, careful and prudent operation but also standardized postoperative management and regular follow-up. Existing radiographic examinations of the ileal graft are more about morphology but less assessment of function (12). Cine MRU and Whitaker test, however, meets the various requirements for monitoring morphology and function of the reconstructed urinary tract. 


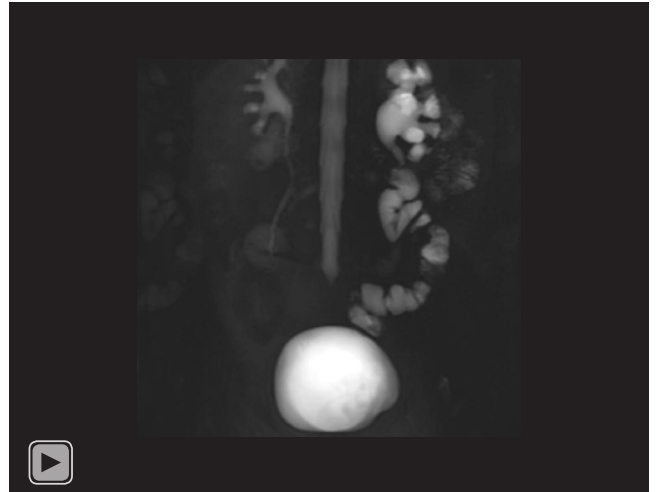

Video 1 Cine MRU of unilateral ileal ureter replacement. MRU, magnetic resonance urography.

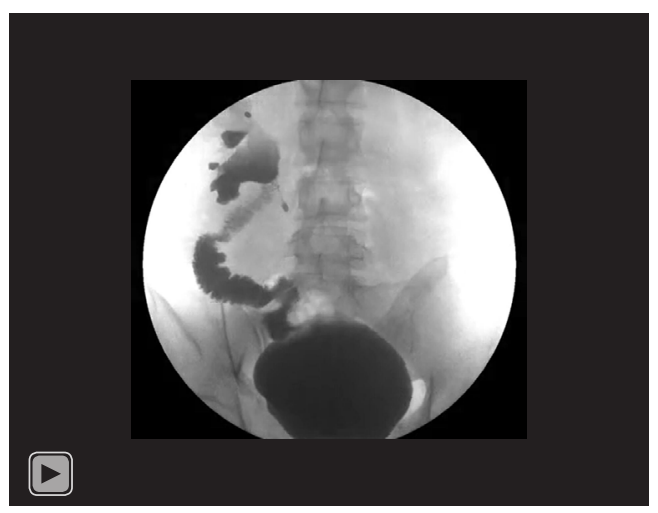

Video 3 Perfusion imaging of unilateral ileal ureter replacement in Whitaker test.

Cine MRI has previously been proved useful to examine gastrointestinal tract motility disorder in a noninvasive way (13-16). Studies have investigated cine MRI in patients with inflammatory bowel disease, pseudo-obstruction, and functional bowel disorders, and the results were helpful (17). On the other hand, gastrointestinal barium radiography is a common method to check digestive system diseases, and it can observe gastrointestinal motility (18). Therefore, it is reasonable to believe that cine MRU and imaging urodynamics are valuable for evaluating the function of ileal graft after ileal ureter replacement.

Occasionally, high resolution of MRU found that the anastomosis was narrow, or the wall was thickened. Meanwhile, the contrast agent passed well and the image was unobstructed in the Whitaker test. Thus, such morphological abnormalities were likely to be caused by postoperative changes and did not require excessive

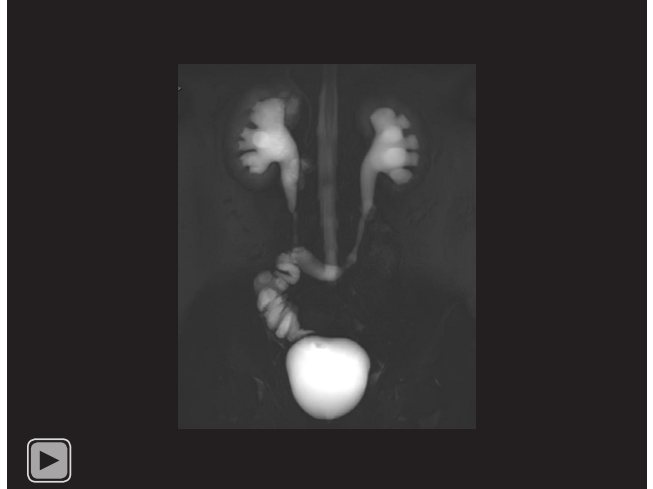

Video 2 Cine MRU of bilateral ileal ureter replacement. MRU, magnetic resonance urography.

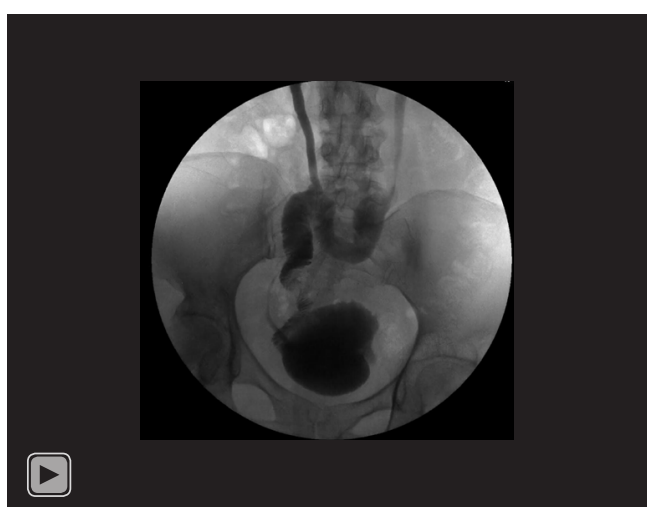

Video 4 Perfusion imaging of bilateral ileal ureter replacement in Whitaker test.

treatment. In addition, MRU suggested anastomotic stenosis or inflammation in 2 patients, and poor imaging was observed in the Whitaker test at the same time. These findings suggested poor drainage of urine, which required close surveillance or even intervention.

Patients with elevated pressure in the Whitaker test must be taken seriously $(8,19,20)$. In the high-pressure group, two patients had the renal pelvis pressure increased and then fell to normal, which indicated that the function of the reconstructed upper urinary tract was slightly worse, while it could still adjust and compensate itself. In another patient, the contraction of the bowel increased significantly, and the renal pelvic pressure and pressure difference also increased, which suggested a mechanical obstruction. Thus, further treatment was recommended.

Cine MRU and Whitaker test can reveal clinically meaningful structural abnormalities of the reconstructed 

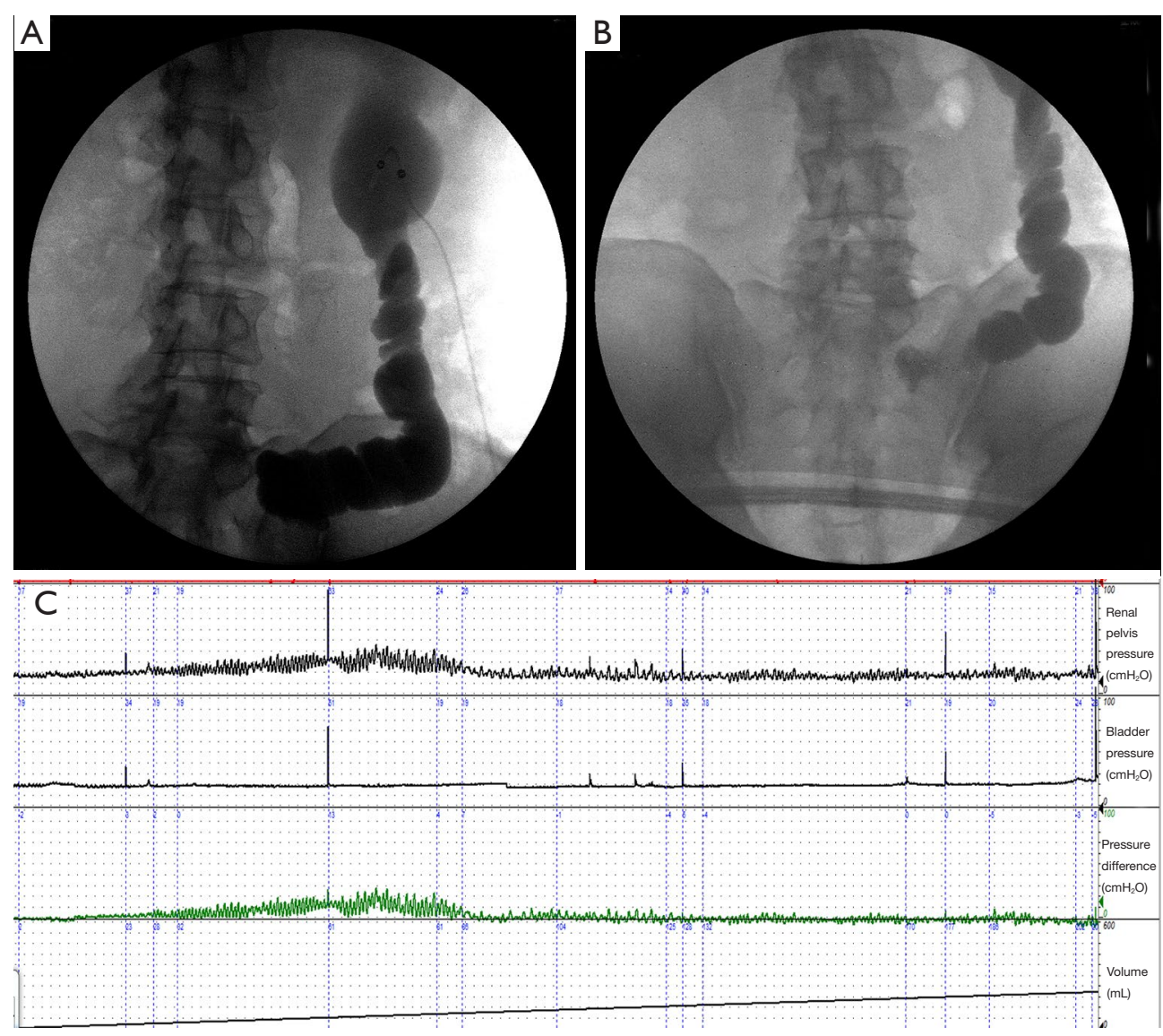

Figure 4 Abnormal results in Whitaker test. (A) Hold-up of contrast medium in ileal graft. (B) Poor imaging at lower ureter. (C) Elevated pressure during perfusion.

upper urinary tract. The Whitaker test evaluates obstruction along the entire length of the reconstructed urinary tract by sensitive pressure changes. In contrast, cine MRU focuses on the peristalsis of the intestine and the result of peristalsis (ureteral jets). Seeing the ureteral jets is strong evidence for the reconstruction of urinary tract patency. In addition, the conventional T1WI, T2WI sequence can evaluate the entire ileal ureter, especially proximal and distal anastomoses. Therefore, the Whitaker test is intuitive, while MRU requires physicians to have a certain foundation of imaging and MRI reading.

Rendering dynamic images, cine MRU and Whitaker test could provide quantitative assessments to offer a comprehensive functional assessment of the reconstructed urinary tract after ileal ureter replacement. In this study, the peristalsis of the elevated pressure group is more active than that of the normal pressure group, but the peristaltic efficiency is lower. Physiologically, the normal ureter contracts until the lumen is closed to form a bolus, allowing transport urine downward (21). However, the diameter of ileal graft is wide, and the lumen cannot be completely closed when contracted. In our preliminary experience, the mean amplitude was $9.29 \mathrm{~mm}$, and the median contraction ratio was 0.607 . It is not enough to evaluate the impetus alone since these active peristalses may not really play an effective role.

Taking the number of peristalsis waves of ileal graft as a reference, the ratio of ureteral jets reflects the effective peristalsis that successfully transports urine. Physiological ureteral peristaltic frequency is $2-6$ times per minute $(22,23)$. The ileal graft had a similar number of peristalses in our patients. The peristalsis efficiency was $100 \%$ in 12 patients, which proves that each peristalsis of ileal graft transported a certain amount of urine downward and the reconstructed upper urinary tract was unobstructed. The peristalsis efficiency was less than 0.5 in another 7 patients. Under this 
Table 2 The detailed evaluation of ileal graft in dynamic MRU

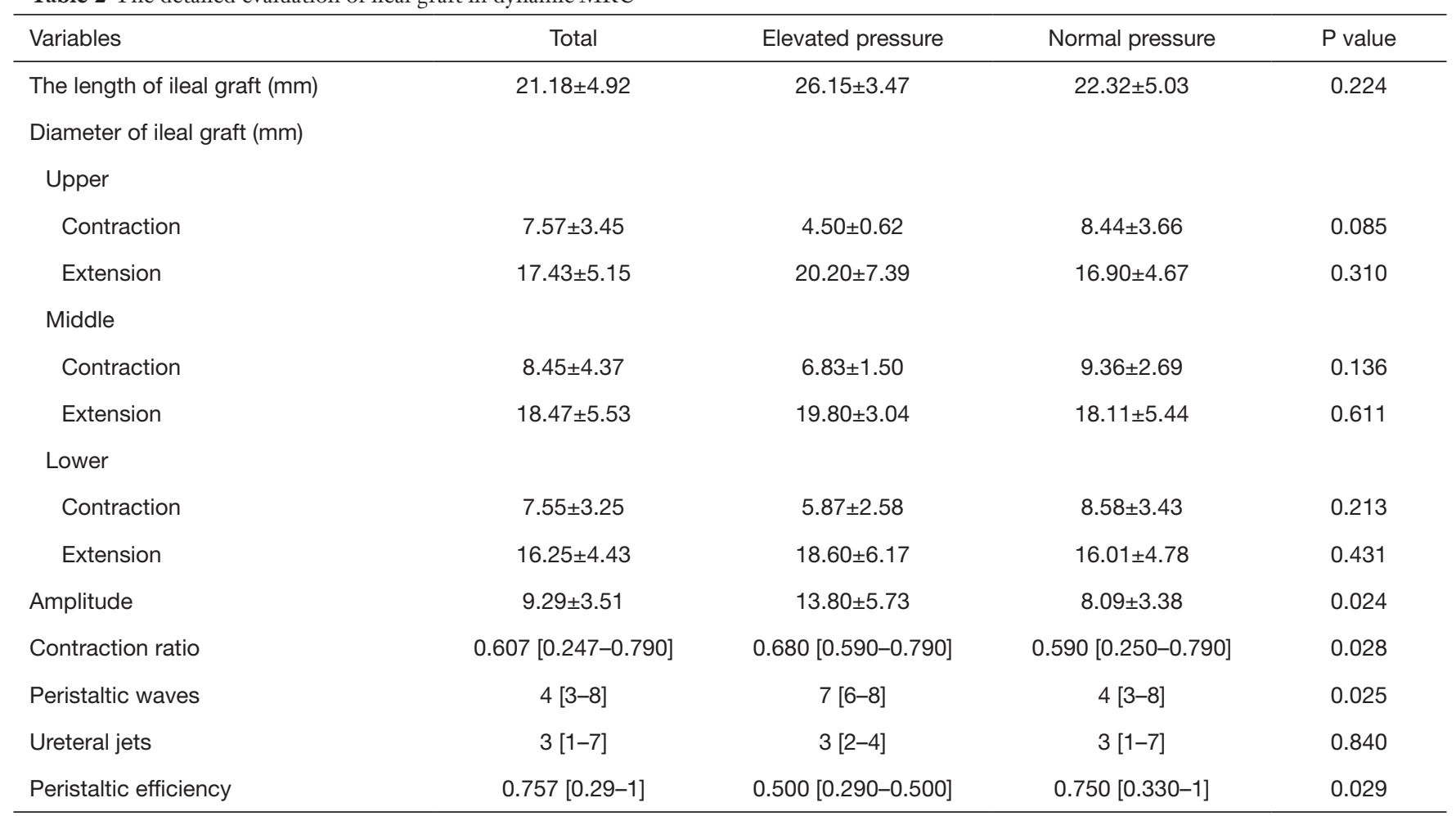

MRU, magnetic resonance urography.

circumstance, obstruction should be suspected no matter what amplitude and contraction ratio were. No jets at all could be a sign of obstruction.

Cine MRU and Whitaker test can also assess the regurgitation of the reconstructed upper urinary tract. Four patients had a pressure difference lower than $0 \mathrm{cmH}_{2} \mathrm{O}$ during the perfusion. A possibility of reflux should be suspected. Cine MRU found no structure anomalies in the distal nipple valve of anti-reflux, and the peristaltic direction of the ileal graft was confirmed as isoperistalsis in all patients. It might be that the large intestinal lumen increased the space in which urine could flow, leading to negative pressure measurement.

Our study has some limitations. Firstly, the main limitation was that it was a retrospective study. Secondly, both Whitaker test and cine MRU had problems with time-consuming and complex processes. The quality of the results was related to the degree of patients' cooperation and the control of breath-holding. Thirdly, the results of diuretic renogram were lacking as a reference. At last, the number of cases was limited, and larger prospective studies are needed.

In conclusion, cine MRU combined with Whitaker test has a certain application prospect and value in evaluating the morphology and the function of the reconstructed ureter after ileal ureter replacement. Quantitative assessments provide a comprehensive functional assessment of the reconstructed urinary tract after ileal ureter replacement. In addition, cine MRU and Whitaker test can also assess the regurgitation of the reconstructed upper urinary tract. However, the biggest limitation of the Whitaker test is the dependent on percutaneous nephrostomy. We do not advocate that patients without a nephrostomy tube establish an invasive channel just for this examination. Therefore, we recommend cine MRU after ileal ureter replacement as a routine examination, and the Whitaker test as a supplementary examination to clarify equivocal cases. Further verification in larger samples is needed.

\section{Acknowledgments}

Funding: None. 


\section{Footnote}

Reporting Checklist: The authors have completed the STROBE reporting checklist. Available at https://dx.doi. org/10.21037/tau-21-507

Data Sharing Statement: Available at https://dx.doi. org/10.21037/tau-21-507

Peer Review File: Available at https://dx.doi.org/10.21037/ tau-21-507

Conflicts of Interest: All authors have completed the ICMJE uniform disclosure form (available at https://dx.doi. org/10.21037/tau-21-507). Xuesong Li serves as an unpaid editorial board member of Translational Andrology and Urology. The other authors have no conflicts of interest to declare.

Ethical Statement: The authors are accountable for all aspects of the work in ensuring that questions related to the accuracy or integrity of any part of the work are appropriately investigated and resolved. The Ethics Committee of Peking University First Hospital approved the study protocol (No. 2020-SR-283), and this study has conformed to the provisions of the Declaration of Helsinki (as revised in 2013). Informed consent was obtained from all participants.

Open Access Statement: This is an Open Access article distributed in accordance with the Creative Commons Attribution-NonCommercial-NoDerivs 4.0 International License (CC BY-NC-ND 4.0), which permits the noncommercial replication and distribution of the article with the strict proviso that no changes or edits are made and the original work is properly cited (including links to both the formal publication through the relevant DOI and the license). See: https://creativecommons.org/licenses/by-nc-nd/4.0/.

\section{References}

1. Hansen MH, Hayn M, Murray P. The Use of Bowel in Urologic Reconstructive Surgery. Surg Clin North Am 2016;96:567-82.

2. Armatys SA, Mellon MJ, Beck SD, et al. Use of ileum as ureteral replacement in urological reconstruction. J Urol 2009;181:177-81.

3. Wolff B, Chartier-Kastler E, Mozer P, et al. Long-term functional outcomes after ileal ureter substitution: a singlecenter experience. Urology 2011;78:692-5.

4. Jeong IG, Han KS, Park SH, et al. Ileal Augmentation Cystoplasty Combined with Ileal Ureter Replacement After Radical Treatment for Cervical Cancer. Ann Surg Oncol 2016;23:1646-52.

5. Leyendecker JR, Barnes CE, Zagoria RJ. MR urography: techniques and clinical applications. Radiographics 2008;28:23-46; discussion 46-7.

6. Wnorowski AM, Guglielmo FF, Mitchell DG. How to perform and interpret cine MR enterography. J Magn Reson Imaging 2015;42:1180-9.

7. Whitaker RH. Methods of assessing obstruction in dilated ureters. Br J Urol 1973;45:15-22.

8. Lupton EW, George NJ. The Whitaker test: 35 years on. BJU Int 2010;105:94-100.

9. Zhong W, Hong P, Ding G, et al. Technical considerations and outcomes for ileal ureter replacement: a retrospective study in China. BMC Surg 2019;19:9.

10. Yang Y, Li X, Xiao Y, et al. A modified Whitaker test (upper urinary tract videourodynamics) using for evaluating complex upper urinary tract reconstruction surgical effect. Transl Androl Urol 2021;10:336-44.

11. Zhu WJ, Ma MM, Zheng MM, et al. Cine magnetic resonance urography for postoperative evaluation of reconstructive urinary tract after ileal ureter substitution: initial experience. Clin Radiol 2020;75:480.e1-9.

12. Bonapace ES, Maurer AH, Davidoff S, et al. Whole gut transit scintigraphy in the clinical evaluation of patients with upper and lower gastrointestinal symptoms. Am J Gastroenterol 2000;95:2838-47.

13. van Rijn KL, Bredenoord AJ, Smout AJPM, et al. Fasted and fed small bowel motility patterns at cine-MRI in chronic intestinal pseudo-obstruction. Neurogastroenterol Motil 2021;33:e14062.

14. Ajaj W, Goehde SC, Papanikolaou N, et al. Real time high resolution magnetic resonance imaging for the assessment of gastric motility disorders. Gut 2004;53:1256-61.

15. Froehlich JM, Patak MA, von Weymarn C, et al. Small bowel motility assessment with magnetic resonance imaging. J Magn Reson Imaging 2005;21:370-5.

16. Fuyuki A, Ohkubo H, Higurashi T, et al. Clinical importance of cine-MRI assessment of small bowel motility in patients with chronic intestinal pseudoobstruction: a retrospective study of 33 patients. J Gastroenterol 2017;52:577-84.

17. de Jonge CS, Smout AJPM, Nederveen AJ, et al. Evaluation of gastrointestinal motility with MRI: Advances, 
challenges and opportunities. Neurogastroenterol Motil 2018. doi: 10.1111/nmo.13257.

18. Szarka LA, Camilleri M. Methods for measurement of gastric motility. Am J Physiol Gastrointest Liver Physiol 2009;296:G461-75.

19. Gill B, Levitt S, Kogan S, et al. The dilated urinary tract in children. Prospective analysis with correlation of radiological, isotope, pressure perfusion and surgical findings. Br J Urol 1988;61:413-9.

Cite this article as: Li X, Wang X, Li T, Zhu W, Ma M, Yang K, Li Z, Wu J, Wang H, Liao L, Li X, Zhou L. Cine magnetic resonance urography and Whitaker test: dynamic visualized and quantified tools in ileal ureter replacement. Transl Androl Urol 2021;10(11):4110-4119. doi: 10.21037/tau-21-507
20. Johnston RB, Porter C. The Whitaker test. Urol J 2014;11:1727-30.

21. Saeki H, Morita T, Weiss RM, et al. The role of ureteral peristaltic rate and bolus volume on increasing urine flow. Urol Int 1986;41:174-9.

22. Edmond P, Ross JA, Kirkland IS. Human ureteral peristalsis. J Urol 1970;104:670-4.

23. Boyarsky S. Behaviour of the Human Ureter in Health and Disease. JAMA 1973;225:1251. 
Table S1 Detailed quantification and imaging combining cine MRU and Whitaker test

\begin{tabular}{|c|c|c|c|c|c|c|c|c|c|c|c|c|c|c|c|}
\hline \multirow{2}{*}{ Patient } & \multicolumn{3}{|c|}{ Caliber of the ileal graft $\left(\mathrm{A}^{\dagger} / \mathrm{B}^{\ddagger}\right)$} & \multirow{2}{*}{$\begin{array}{l}\text { Length of ileal } \\
-\quad \text { graft }(\mathrm{mm})\end{array}$} & \multirow{2}{*}{$\begin{array}{c}\text { Mean } \\
\text { Amplitude } \\
(\mathrm{mm})\end{array}$} & \multirow{2}{*}{$\begin{array}{l}\text { Mean } \\
\text { contraction } \\
\text { ratio }\end{array}$} & \multirow{2}{*}{$\begin{array}{l}\text { Urine } \\
\text { jets (n) }\end{array}$} & \multirow{2}{*}{$\begin{array}{l}\text { Peristaltic } \\
\text { waves }\end{array}$} & \multirow{2}{*}{$\begin{array}{l}\text { Pressure } \\
\text { difference } \\
\left(\mathrm{cmH}_{2} \mathrm{O}\right)\end{array}$} & \multirow{2}{*}{$\begin{array}{l}\text { Pelvis } \\
\text { pressure } \\
\left(\mathrm{cmH}_{2} \mathrm{O}\right)\end{array}$} & \multirow{2}{*}{$\begin{array}{l}\text { Perfusion } \\
\text { volume } \\
\text { (mL) }\end{array}$} & \multirow{2}{*}{$\begin{array}{c}\text { Final perfusion } \\
\text { volume } \\
(\mathrm{mL})\end{array}$} & \multicolumn{2}{|c|}{ Imaging in MRU } & \multirow{2}{*}{$\begin{array}{l}\text { Imaging in } \\
\text { Whitaker } \\
\text { test }\end{array}$} \\
\hline & Upper (mm) & Middle $(\mathrm{mm})$ & Lower (mm) & & & & & & & & & & Peristalsis & Anastomosis & \\
\hline 1 & $4.30 / 28.60$ & $8.50 / 22.50$ & $3.10 / 24.50$ & 28.33 & 19.90 & 0.79 & 3 & 6 & 40 & 57 & NA & 55 & Regular & $\begin{array}{l}\text { Narrow and } \\
\text { local wall } \\
\text { thickening }\end{array}$ & UPJ narrow \\
\hline 2 & $4.00 / 14.70$ & $5.60 / 16.50$ & $8.20 / 12.20$ & 27.97 & 9.87 & 0.62 & 2 & 7 & -6 & 54 & 113 & 211 & Regular & Bilateral narrow & Clear \\
\hline 3 & $5.20 / 17.30$ & $6.40 / 20.40$ & $6.30 / 19.10$ & 22.14 & 13.0 & 0.69 & 4 & 8 & -5 & 26 & 71 & 205 & Regular & Inflammation & $\begin{array}{l}\text { Poor imaging } \\
\text { at lower ureter }\end{array}$ \\
\hline 4 & $5.70 / 28.70$ & $7.30 / 18.00$ & $4.50 / 8.60$ & 14.47 & 12.60 & 0.68 & 2 & 3 & -24 & Normal & - & 53 & Slow & Normal & Clear \\
\hline 5 & $6.70 / 14.80$ & $6.90 / 16.80$ & $4.70 / 21.50$ & 14.44 & 11.60 & 0.66 & 6 & 6 & -3 & Normal & - & 243 & Regular & Normal & Clear \\
\hline 6 & $6.80 / 15.10$ & $11.40 / 22.70$ & $11.50 / 20.20$ & 22.53 & 9.43 & 0.49 & 4 & 4 & 3 & Normal & - & 307.8 & Slow & Slight narrow & Clear \\
\hline 7 & $4.20 / 11.60$ & $4.70 / 10.80$ & $2.50 / 9.40$ & 17.06 & 6.80 & 0.64 & 7 & 7 & 5 & Normal & - & 170 & Regular & Slight narrow & Clear \\
\hline 8 & $3.00 / 14.40$ & $9.10 / 25.60$ & $7.20 / 14.10$ & 28.20 & 11.60 & 0.64 & 6 & 8 & 6 & Normal & - & 197 & Regular & Normal & Clear \\
\hline 9 & $9.30 / 18.60$ & $8.80 / 17.40$ & $3.60 / 12.50$ & 25.77 & 8.93 & 0.55 & 4 & 5 & 1 & Normal & - & 126 & Regular & Normal & Clear \\
\hline 10 & $4.23 / 11.96$ & $6.64 / 16.78$ & $8.09 / 15.44$ & 19.51 & 8.41 & 0.57 & 2 & 4 & -2 & Normal & - & 120 & Regular & Normal & Clear \\
\hline 11 & $5.65 / 22.00$ & $9.21 / 24.72$ & $11.42 / 25.70$ & 30.88 & 15.38 & 0.64 & 5 & 5 & 1 & Normal & - & 43 & Regular & Normal & Clear \\
\hline 12 & $8.10 / 17.79$ & $12.77 / 28.71$ & NA & 19.76 & 8.54 & 0.55 & 2 & 3 & -2 & Normal & - & 22 & Regular & Normal & Clear \\
\hline 13 & $16.46 / 22.34$ & $10.90 / 19.71$ & $11.07 / 19.33$ & 29.11 & 7.65 & 0.37 & NA & 3 & 3 & Normal & - & 120 & Regular & Normal & Clear \\
\hline 14 & $7.92 / 10.41$ & $8.92 / 11.81$ & $11.07 / 14.86$ & 21.74 & 3.06 & 0.25 & 1 & 3 & 1 & Normal & - & 160 & Regular & Normal & Clear \\
\hline 15 & $11.92 / 20.80$ & $12.69 / 18.07$ & $11.43 / 18.84$ & 25.69 & 7.22 & 0.38 & NA & 4 & -1 & Normal & - & 100 & Regular & Normal & Clear \\
\hline 16 & $11.56 / 17.94$ & $15.38 / 22.28$ & $8.93 / 18.74$ & 27.26 & 7.70 & 0.39 & 2 & 4 & 2 & Normal & - & 120 & Regular & Normal & Clear \\
\hline 17 & $14.49 / 17.44$ & $8.30 / 17.85$ & $10.81 / 17.94$ & 25.36 & 6.54 & 0.37 & 4 & 6 & 3 & Normal & - & 140 & Regular & Normal & Clear \\
\hline 18 & $7.92 / 12.51$ & $6.59 / 8.91$ & 6.19/9.06 & 15.67 & 3.26 & 0.32 & 2 & 3 & 14 & Normal & - & 120 & Regular & Normal & Clear \\
\hline 19 & $10.34 / 16.46$ & $10.50 / 14.56$ & $14.02 / 17.24$ & 22.81 & 4.47 & 0.28 & NA & 4 & 10 & Normal & - & 110 & Regular & Normal & Clear \\
\hline 20 & $9.26 / 14.44$ & $9.00 / 13.10$ & $10.28 / 13.89$ & 19.46 & 4.30 & 0.31 & 3 & 4 & 1 & Normal & - & 140 & Regular & Normal & Clear \\
\hline
\end{tabular}

${ }^{\dagger}$, diameter of maximum contraction; ${ }^{\ddagger}$, diameter of maximum extension. MRU, magnetic resonance urography. 